INRA Prod. Anim., 1994,7 (2), 71 - 84 .
C. JEAN-BLAIN

L. ALVES de OLIVEIRA

INRA Unité Physio-pathologie du rumen ENV 1, avenue Bourgelat, BP 83 - 69280 Marcy-l'Etoile

\section{Aspects physio- pathologiques de la thiamine (vitamine B1) chez les ruminants}

La vitamine $\mathrm{B} 1$ chez les ruminants a retenu l'attention des nutritionnistes depuis la mise en évidence en 1961 d'un syndrome nerveux carentiel décrit sous le nom de Nécrose du Cortex Cérébral. Des travaux canadiens et américains récents semblent remettre en cause l'étiologie carentielle. Qu'en est-il réellement? Par ailleurs, la synthèse de vitamine B1 en l'absence de toute manifestation clinique de carence est elle toujours optimale ? Cette revue tente de faire le point sur ces différentes interrogations.

L'absence de dépendance alimentaire des ruminants pour les vitamines du groupe B a été suspectée peu de temps après leur découverte au début $\mathrm{du} 20^{\mathrm{imc}}$ siècle. Cette suspicion reçoit très rapidement une confirmation expérimentale. Ainsi la distribution d'extraits de jus de rumen à des rats recevant un régime semi-synthétique, pauvre en vitamines du groupe B, fait disparaitre les signes de carence qu'ils présentent (Bechdel et al 1928). Avec un régime pauvre en vitamines $B$, la quantité de vitamine retrouvée dans le rumen est supérieure à celle qui est apportée par l'ali-

\section{Résumé}

Cette revue envisage d'abord la production microbienne, l'absorption et l'utilisation métabolique de la thiamine chez le ruminant sain ainsi que les effets d'une synthèse insuffisante de thiamine sur l'activité microbienne du rumen. Elle fait ensuite une analyse critique des données à la fois classiques et récentes concernant l'étiologie et la pathogénie de la nécrose du cortex cérébral (NCC). Les facteurs impliqués dans la synthèse et la dégradation de la thiamine dans les conditions normales d'alimentation sont relativement bien connus. Il n'en va pas de même des facteurs qui sont responsables de la diminution de la production nette de thiamine dans le rumen ou de son absorption, dans des conditions de sous- ou de sur-alimentation ou avec des régimes déséquilibrés. La pathogénie classique de la nécrose du cortex met en jeu la surproduction de thiaminases de type I. Cette théorie n'est cependant pas entièrement satisfaisante; en effet la micropopulation du rumen a normalement une activité thiaminasique globale très faible qu'il est impossible de faire évoluer expérimentalement à l'heure actuelle. L'excès de soufre dans la ration peut également induire l'apparition de lésions typiques de NCC. Dans ce cas la relation avec la thiamine n'est pas évidente. Il est probable que l'on a affaire à deux étiologies complètement différentes qui s'expriment par le même type de lésions. mentation (McElroy et Goss 1941). L'utilisation de régimes synthétiques dépourvus de vitamines du groupe B (Agrawala et al 1953, Virtanen 1967) apporte la preuve rigoureuse que les ruminants peuvent synthétiser l'ensemble des vitamines appartenant à ce groupe. Ultérieurement la plupart des essais de terrain démontrent que l'adjonction de vitamines du groupe B n'a aucun effet sur les performances et l'état de santé des animaux.

En ce qui concerne la thiamine (vitamine B1) ce concept de non dépendance alimentaire du ruminant aux vitamines du groupe B s'est relativisé à la suite de la mise en évidence de manifestations carentielles décrites sous le nom de nécrose du cortex cérébral ou polioencéphalomalacie. Si l'action curative de la vitamine B1 sur ce syndrome a pu être démontrée avec précision, par contre le mécanisme de son apparition n'a jamais pu être établi de façon certaine (Loew et al 1975). A la suite des travaux poursuivis actuellement par les auteurs canadiens et américains, une autre étiologie, en relation avec les excès de soufre dans la ration, se fait jour. Des études sont encore nécessaires pour clarifier le problème et préciser les éventuelles interdépendances entre l'excès de soufre et une déficience en thiamine.

Par ailleurs en l'absence de toute pathologie, un supplément alimentaire de vitamine B1 peut, dans certains cas, optimiser l'activité 
fermentaire du rumen (régimes à base de fourrages pauvres, changements brutaux de régimes avec des régimes très riches en concentrés). Nous tenterons d'examiner dans cet article les différents aspects physiologiques et pathologiques de la nutrition vitaminique $\mathrm{B} 1$ et ses relations éventuelles avec le métabolisme du soufre.

\section{1 / Métabolisme de la thiamine chez les ruminants}

\section{1 / Rappel sur le rôle physiologique de la thiamine (figure 1)}

La thiamine existe dans l'organisme soit sous forme libre, soit sous forme d'esters phosphoriques, thiamine monophosphate (TMP), thiamine pyrophosphate (TPP) et thiamine triphosphate (TTP) (figure 2). La fonction du TTP n'est pas encore connue avec exactitude. Le TMP constitue un intermédiaire dans la dégradation du TPP en thiamine libre. Le TPP ou thiamine pyrophosphate constitue la forme biologiquement active la plus répandue dans l'organisme. Le TPP intervient comme coenzyme des décarboxylases dans les réactions de décarboxylation irréversibles des acides alphacétoniques (pyruvate, alphacétoglutarate, et également 3-alphacétoacides dérivés des acides aminés ramifiés). Il constitue également le coenzyme des transcétolases qui transferent un groupement cétol dicarboné d'un pentose phosphate sur un sucre en $\mathrm{C} 4$ ou en C5. Les transcétolases interviennent dans le cycle des pentoses dans lequel leur activité constitue une étape limitante. Le dosage des transcétolases est donc un bon moyen d'appréciation de l'état carentiel en vitamine B1 dans toutes les espèces animales. L'activité enzymatique multiple du TPP lui confère un rôle important dans le métabolisme énergétique du glucose, dans le fonctionnement du cycle de Krebs, dans la dégradation oxydative des acides aminés : glutamate, acides aminés ramifiés et, à un moindre degré, acides aminés dont la dégradation aboutit au pyruvate (alanine, cystéine, glycocolle, sérine, thréonine), lorsque le pyruvate n'est pas utilisé pour la néoglucogenèse. Chez les ruminants, le cerveau ne peut pas utiliser les corps cétoniques au cours du jeûne, même après une période de transition, contrairement à d'autres espèces. L'intégrité du tissu nerveux est donc toujours liée à une oxydation complète du glucose. Par ailleurs, le cycle des pentoses joue un très grand rôle au niveau de la mamelle pour produire du NADP réduit, nécessaire à la synthèse des acides gras du lait.

En dehors de ce rôle fondamental de coenzyme, la thiamine joue un rôle direct dans le fonctionnement du système nerveux, bien que son mode d'action dans ce domaine ne soit pas encore très bien connu (Haas 1988). Elle intervient dans la conduction de l'influx nerveux et dans le fonctionnement des synapses neuromusculaires, ainsi que dans le métabolisme de plusieurs neuro-transmetteurs au niveau du tissu cérébral.

\section{2 / Absorption}

La thiamine apportée par la ration ou synthétisée dans le rumen est absorbée dans l'intestin grêle, principalement au niveau du jéjunum. En utilisant des techniques très variées, in vitro : incubation de muqueuse isolée de rumen en chambre de Ussing (Höller et al 1977) et in vivo : rumen lavé isolé (Höller et al 1979), transit digestif croisé sur des vaches porteuses de fistules du rumen et de canules réentrantes du duodénum (Steinberg et al 1977) les chercheurs de l'Ecole Vétérinaire de Hanovre ont démontré l'imperméabilité com-

Figure 1. Rôles physiologiques de la thiamine.

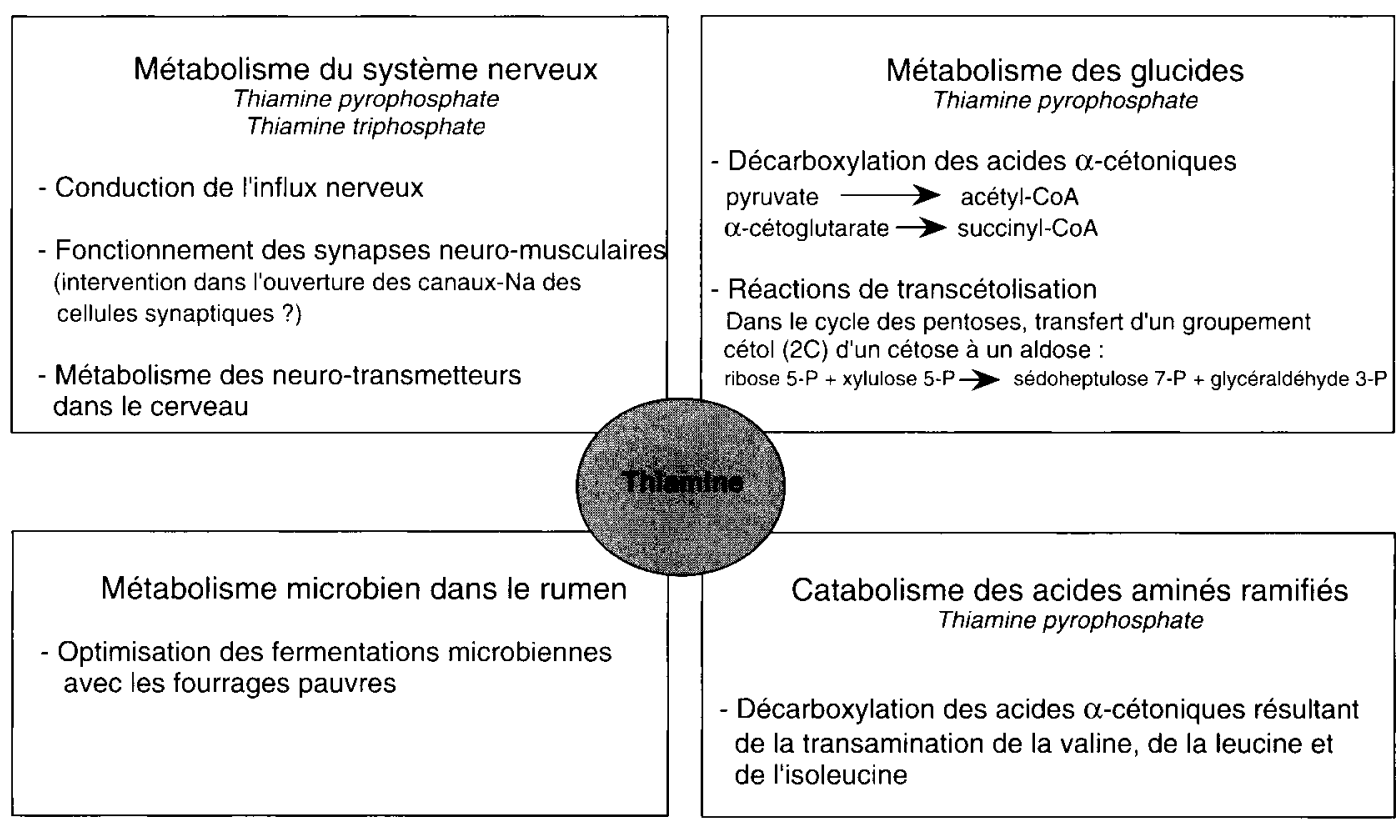


Figure 2. Interconversions entre la thiamine et ses dérivés phosphorylés.

Thiamine libre<smiles>C/C=[N+](/C)Cc1cnc(C)nc1N</smiles><smiles>Cc1ccsc1CCO</smiles>

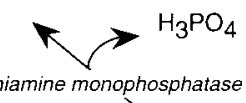
Thiamine monophosphate

thiamine monophosphatase<smiles>Cc1ncc(C[n+]2csc(CCOP(=O)(O)O)c2C)c(N)n1</smiles>

Thiamine pyrophosphate<smiles>Cc1ncc(C[n+]2csc(CCOP(=O)(O)OP(=O)(O)O)c2C)c(N)n1</smiles><smiles>CCC(C)C(C)C(C)C(C)C</smiles>

Thiamine triphosphate<smiles>Cc1ncc(C[n+]2csc(CCOP(=O)(O)OP(=O)(O)OP(=O)(O)O)c2C)c(N)n1</smiles>

plète de la paroi du rumen à la vitamine B1. La possibilité également d'une absorption de thiamine par le gros intestin a été étudiée, une certaine quantité de thiamine étant en effet synthétisée par les bactéries dans cet organe. Une perfusion de thiamine dans le gros intestin préalablement lavé est récupérée à $87 \%$ dans les fèces (Höller et al 1982). L'absorption reste donc faible dans cette portion du tube digestif. Son rôle sur l'utilisation de la thiamine alimentaire ou synthétisée dans le rumen est négligeable si l'on considère que la quasi totalité de cette thiamine est absorbée dans l'intestin grêle.

L'absorption de la thiamine se fait donc essentiellement dans le jéjunum grâce à un mécanisme d'absorption actif, saturable, nécessitant $\mathrm{Na}^{+}$et la participation de l'ATPase $\mathrm{Na}^{+} / \mathrm{K}^{+}$dépendante. La thiamine est phospho- rylée dans la muqueuse jéjunale en TPP. A très forte concentration elle peut cependant diffuser passivement à travers la muqueuse jéjunale.

\section{3 / Stockage et répartition dans l'organisme}

La thiamine n'est pas stockée dans l'organisme comme c'est le cas pour les vitamines liposolubles. Elle se répartit dans tous les tissus de façon inégale. Chez le boeuf et le mouton la teneur des différents tissus varie entre 0,5 et $5 \mu \mathrm{g} / \mathrm{g}$ (muscle $<$ cerveau $<$ rein $<$ foie $<$ coeur).

Dans le sang total, la teneur moyenne en thiamine est de $120 \mathrm{nmol} / \mathrm{l}$ chez le bœuf et le mouton au pâturage, avec une fourchette de 75 - $184 \mathrm{nmol} /$ (Hill et al 1988). Des valeurs 
moyennes analogues de $140 \mathrm{nmol} / \mathrm{l}$, avec une dispersion encore plus grande (10-240), ont été relevées au Canada sur 221 animaux lors d'une enquête portant sur 50 exploitations (Olkowski et al 1991a). L'administration par injection d'une dose élevée de thiamine peut transitoirement décupler ces valeurs. Compte tenu des fluctuations individuelles importantes, la détermination du taux de thiamine dans le sang total ne permettra d'apprécier le niveau de couverture des besoins que si elle est réalisée sur un nombre d'animaux importants.

La thiamine est répartie inégalement entre les hématies $(75 \%)$ et le plasma $(25 \%)$. Chez l'homme, $85 \%$ de la thiamine du sang total et $96 \%$ de la thiamine des globules rouges est sous forme de TPP, 2 et $3 \%$ respectivement sous forme de TTP. Le plasma contient uniquement du TMP et de la thiamine libre (Egi et al 1992). Il n'existe pas de données correspondantes pour les ruminants.

Des études cinétiques d'élimination de thiamine radioactive ont permis de décrire la répartition de thiamine dans l'organisme dans trois compartiments dynamiques (Breves et al 1982). Le transport intracellulaire de thiamine dans les cellules a été étudié dans les hépatocytes ; il se fait par un antiport thiamine $/ \mathrm{H}^{+}$ (Moseley et al 1992).

\section{4 / Excrétion et élimination dans le lait}

L'excrétion de la thiamine et de ses métabolites se fait à la fois par l'urine et par les fèces. L'excrétion fécale ne concerne pratiquement que la thiamine synthétisée par les bactéries du gros intestin.

L'étude de l'excrétion urinaire de thiamine ou, le cas échéant, de ses métabolites, peut-elle avoir une valeur pratique pour l'exploration de l'état de nutrition vitaminique de l'organisme? Chez l'homme,en cas de carence d'apport, l'excrétion urinaire diminue de moitié au 10ème jour de carence. Lorsque les symptômes de polynévrite apparaissent, la thiamine urinaire tombe à une valeur presque nulle. En cas d'apport très faible, la teneur de l'urine atteint une valeur nulle, mais l'urine contient les métabolites de la thiamine représentés par des dérivés de la pyrimidine et du thiazole (Ziporin et al 1985). Une détermination unique de la B1 dans les urines n'a pas de valeur diagnostique ; elle ne renseigne pas en effet sur le stock de thiamine ou sur sa disponibilité, dans la mesure où cette excrétion peut être influencée par un apport extérieur important et ponctuel.

L'excrétion fécale reste importante en cas de carence, ce qui montre que la thiamine synthétisée dans le colon n'est pas ou très peu absorbée. Chez le mouton, l'excrétion urinaire de thiamine est influencée par la quantité de thiamine ingérée si cette dernière est protégée (Inglis et al 1989) ; dans le cas contraire, l'excès de thiamine est détruit dans le rumen par les bactéries. Par contre l'administration parentérale de thiamine augmente l'élimination urinaire. Par ailleurs, l'administration en continu de fortes doses de thiamine par voie parentéra- le induit d'abord une forte élimination urinaire qui diminue ensuite avec le temps, ce qui montre soit qu'il s'établit un stockage de vitamine dans les tissus, plus important que dans les conditions normales d'apport, ou que, plus vraisemblablement, il existe une adaptation métabolique qui provoque la destruction de l'excès de thiamine administrée.

L'élimination de thiamine dans le lait est peu variable (teneur du lait frais : de 0,3 à 0,7 $\mu \mathrm{g}$ par litre). Une sous-alimentation énergétique ou azotée n'a aucune influence sur cette teneur (Kirchgessner et al 1988).

\section{2 / La synthèse microbienne de thiamine chez les ruminants}

\section{1 / Répartition de la thiamine dans le rumen}

La plus grande partie ( 85 à $90 \%$ ) de la thiamine du contenu de rumen se trouve sous forme estérifiée. La teneur en thiamine totale dans le rumen se situe dans une fourchette large de 0,05 à $1,9 \mu \mathrm{g} / \mathrm{g}$ de jus ; elle est minimale $(0,05$ à $0,1 \mu \mathrm{g} / \mathrm{g})$ avec une ration à base d'ensilage de mais, plus importante $(0,2 \mu \mathrm{g} / \mathrm{g})$ avec une ration à base de foin (Massengo 1977 , Candau et Massengo 1982). Les controverses concernant la répartition de la thiamine entre les particules alimentaires, les microorganismes et la phase liquide débarrassée de ses bactéries sont nombreuses et dues probablement à des problèmes méthodologiques : lyses des bactéries, adhérence des bactéries sur particules alimentaires. Des mesures effectuées sur du contenu de rumen de mouton ont montré que la plus grande partie de la thiamine se trouve dans les bactéries.

\section{2 / Mesure de la production de thiamine dans le rumen}

La production de thiamine dans le rumen est en général déterminée par mesure du flux de thiamine dans le duodénum au moyen d'une canule réentrante. La différence entre le flux duodénal et l'apport alimentaire donne la production nette de thiamine dans le rumen (somme synthèse + dégradation microbienne), puisqu'il n'y a pas d'absorption par la paroi du rumen (figure 3).

\section{3 / Influence de l'apport alimentaire de thiamine sur sa synthèse microbienne dans le rumen}

C'est un des plus importants facteurs de variation. Il existe une relation linéaire entre l'apport alimentaire de thiamine et le flux duodénal (Miller et al 1986, Breves et al 1981). Lorsque l'apport alimentaire est faible, voire insignifiant, la synthèse intraruminale de $\mathrm{B} 1$ peut atteindre 20 à 25 fois l'apport alimentaire. Lorsqu'il est très élevé, la production nette est négative. 
Figure 3. Bilan de thiamine dans le tube digestif d'une vache laitière recevant une ration mixte ensilage de maïs, foin, concentrés- (d'après Breves et al 1981).

$$
\begin{aligned}
& \text { Apport de thiamine par la ration } \\
& 52 \mathrm{mg} / \mathrm{j}
\end{aligned}
$$$$
(3,8 \mathrm{mg} / \mathrm{kg} \mathrm{MS} / \mathrm{j})
$$

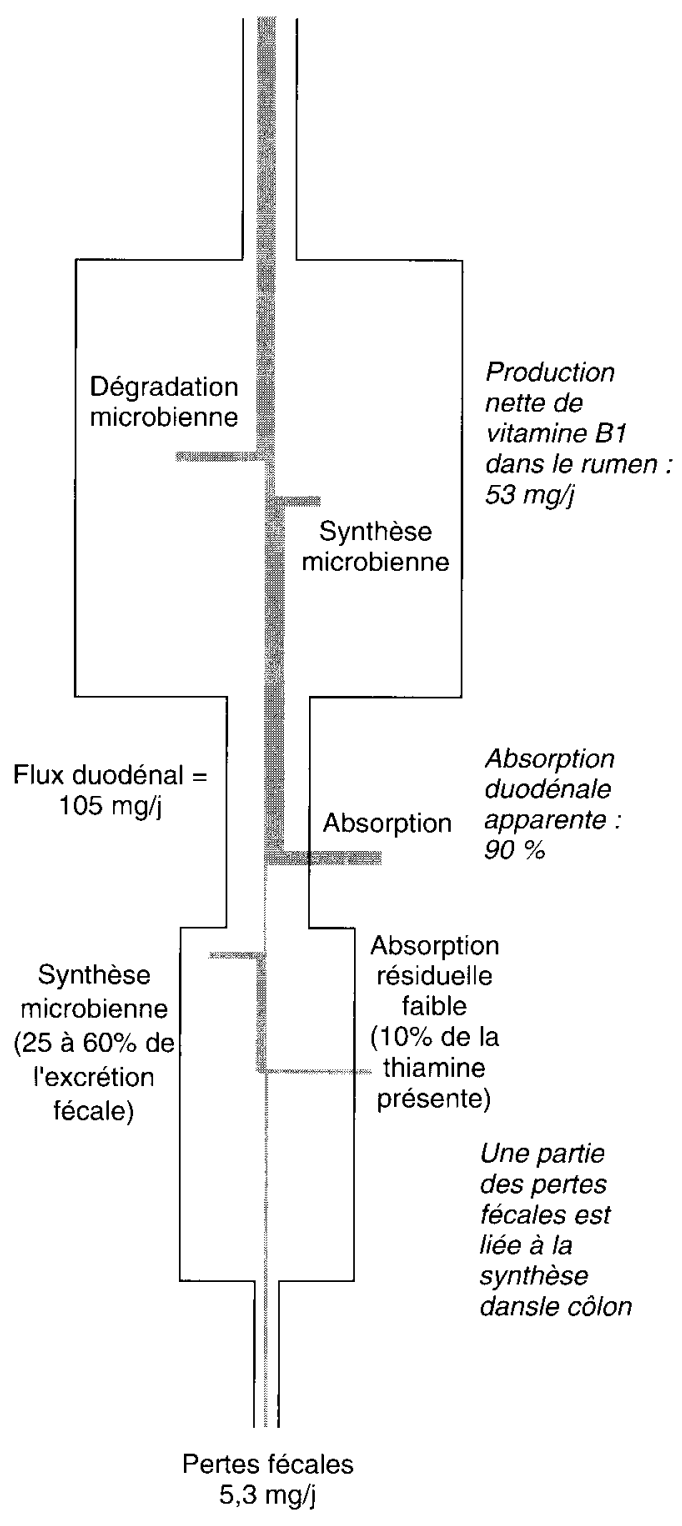

\section{4 / Effet de l'apport azoté de la ration}

L'effet de l'apport azoté (forme, dose d'apport et rythme de distribution) sur le flux duodénal et la synthèse nette de thiamine a été étudié chez la vache laitière recevant une ration classique de foin, d'ensilage de maïs et de concentré (Breves et al 1981). La réduction de l'apport azoté diminue la synthèse nette de thiamine. Par contre, le remplacement d'une partie de l'azote protéique par de l'urée, ou le rythme de distribution, n'ont pas d'effet sur cette synthèse. Il y a une corrélation étroite entre le flux d'azote microbien dans le duodénum et le flux de thiamine.

\section{5 / Effet du niveau d'apport et de la nature des glucides alimentaires}

L'effet sur le flux duodénal de thiamine de différents types de céréales utilisées à un taux très élevé ( $85 \%$ de la ration) a été testé sur des génisses (Miller et al 1986). Tous les régimes ont donné, à l'exception du régime à base de sorgho, une production ruminale nette de thiamine négative, sans relation avec les $\mathrm{pH}$ ruminaux bas induits par ce type de régime. Dans ces expériences, l'apport alimentaire de thiamine était très élevé.

\section{6 / Effet de l'apport de soufre et du rapport $\mathbf{N} / \mathbf{S}$}

Le soufre est nécessaire aux bactéries du rumen pour synthétiser leurs propres protéines. Le rapport N/S est susceptible d'influencer la production de biomasse microbienne et, partant, la production de thiamine. Par ailleurs les études épidémiologiques canadiennes ont montré que des apports excessifs de sulfate constituaient un facteur de risque important pour l'apparition de la nécrose du cortex (Olkowski et al 1991a, Olkowski 1992). Nous n'avons malheureusement que très peu d'informations directes sur l'effet du soufre sur la synthèse de thiamine. Des études in vitro (Bick et al 1978, Olkowski et al 1993) avec des incubations de courte durée ne permettent pas de mettre en évidence une synthèse nette de thiamine. Tout au plus permettent-elles de conclure, et avec beaucoup de prudence, que la perte en thiamine au cours des incubations est plus faible avec des rapports N/S élevés (20 à 30) qu'avec des rapports N/S bas ou moyens (9 à 15). Par ailleurs, in vitro, les sulfites provoquent un clivage de la thiamine en ses deux noyaux constitutifs (Leichter et Joslyn 1969). Il n'est cependant pas évident que cette particularité puisse avoir une conséquence au niveau du rumen, les sulfates étant très rapidement réduits en sulfure et l'étape sulfite étant pratiquement virtuelle.

\section{7 / Effet de l'apport de phosphore}

Un unique travail fait état, paradoxalement, d'une augmentation du pool de thiamine dans le rumen et du flux duodénal lors d'une déplétion en phosphore (Barschdorf 1985), alors que la teneur en thiamine des bactéries est diminuée et que l'on sait que le déficit en phosphore diminue la protéosynthèse. L'augmentation observée serait liée à une diminution de la dégradation bactérienne de la thiamine.

\section{8 / Effet des antibiotiques}

Le monensin diminue la production nette de thiamine dans le réticulo-rumen (Lebzien et al 1986). Cette diminution est liée étroitement à la dépression de la synthèse de protéines microbiennes. Avec des régimes très riches en concentré, l'introduction de monensin ou de chlortétracycline à la dose de $70 \mathrm{mg}$ par jour n'a pas d'effet sur le flux duodénal de thiamine (Miller et al 1986). Par contre une dose de $1 \mathrm{~g}$ par jour tend à diminuer la dégradation de 
thiamine dans le rumen et à diminuer aussi son absorption dans l'intestin grêle.

\section{9 / Mécanismes de régulation de la synthèse microbienne de thiamine}

Les études de bilan précédemment citées montrent que la synthèse nette peut présenter des valeurs négatives, particulièrement lorsque l'apport alimentaire de thiamine est élevé. Ceci implique une modulation de la synthèse bactérienne de thiamine et également des mécanismes de destruction de la thiamine préformée. In vitro, $35 \%$ de la thiamine incubée avec du jus de rumen est dégradée par les bactéries au bout d'une heure (El Hindi 1977). La régulation de la synthèse microbienne de la thiamine n'est abordée que très superficiellement dans des travaux anciens (Newell et Tucker 1966) portant sur Salmonella typhimurium. Dans un milieu riche en $\mathrm{B} 1$ la répression due à l'excès de thiamine s'exerce sur l'enzyme combinant le noyau thiazole et le noyau pyrimidine puis sur les systèmes enzymatiques intervenant dans la synthèse de ces deux composants. In vitro, en fermenteur RUSITEC (travaux non publiés) nous avons pu montrer que la thiamine ajoutée dans le milieu d'incubation est très rapidement absorbée ou détruite par les bactéries, et qu'en même temps, la production nette de thiamine diminue et ceci en l'absence de toute activité thiaminolytique dans le milieu. Les mécanismes de rétroinhibition apparaissent donc comme importants dans la régulation de la production microbienne de thiamine. En ce qui concerne les microorganismes de l'écosystème rumen, l'intervention respective des différents groupes microbiens ainsi que les mécanismes régulateurs mis en jeu sont totalement inconnus. Un seul travail fait état du métabolisme de la thiamine chez différents groupes bactériens isolés à partir du rumen d'animaux sains ou atteints de nécrose du cortex (Haven et al 1983). Chez les animaux atteints de NCC la quasi totalité des bactéries Gram + et - , à l'exception des cocobacilles Gram +, provoquent une destruction de la thiamine, alors que chez les animaux sains les bacilles Gram - produisent une synthèse suffisante pour compenser les destructions dues aux autres groupes bactériens. La fiabilité des résultats obtenus dans ce travail est toutefois mise en doute par le fait qu'une quantité importante de thiamine était rajoutée à chaque milieu de culture ; en outre, la méthode de dosage utilisée ne permet pas de dire si la disparition de la thiamine observée était due à sa destruction ou à sa transformation en ester phosphorique.

La dégradation de la thiamine dans le tube digestif, et en particulier dans le rumen, peut être due à des facteurs thiaminolytiques non enzymatiques ou à des thiaminases. Les deux types de facteurs peuvent exister conjointement ou non dans les aliments ou dans certains végétaux tels que la fougère aigle (Fujita 1954).

De nombreux aliments sont susceptibles d'avoir in vitro une activité antithiamine importante (Höller et al 1976) ; c'est le cas des betteraves fourragères, des feuilles de betterave, des pulpes de sucrerie. Cette activité antithiamine est thermostable, elle n'est pas due à la présence de thiaminases. L'acide caféique et l'hémine ont, in vitro, une activité antithiamine. Ces substances administrées in vivo à des rats ne permettent pas cependant de reproduire un état de carence, du moins aux doses où on les retrouve habituellement dans les aliments (Schaller et al 1977).

L'essentiel de l'action thiaminolytique est attribuée aux thiaminases. Celles-ci sont très largement répandues dans la nature ; elles sont soit produites par des bactéries, soit contenues dans certains tissus végétaux ou animaux. La bactérie productrice de thiaminase la mieux connue est Bacillus thiaminolyticus isolée à partir de l'intestin humain. Ultérieurement, deux autres micro-organismes producteurs de thiaminases ont été isolés à partir de fêces humaines, Bacillus aneurinolyticus et Clostridium thiaminolyticum. On retrouve une activité thiaminasique dans les viscères et la chair de différents poissons, dans certains mollusques et crustacés, dans les rhizomes et les frondes de fougère aigle (Fujita 1954). Du point de vue du mécanisme d'action il existe deux types de thiaminases (figure 4), les thiaminases de type I (EC 3.5.1.2.) et de type II (EC 3.5.99.2). Les thiaminases de type II sont des hydrolases qui coupent la molécule de thiamine entre le pont méthylène et l'ammonium quaternaire du noyau thiazole. Les thiaminases de type I sont des transférases qui vont fixer un co-substrat sur la partie pyrimidique à la place du noyau thiazole qui est libéré. Les thiaminases de type I ne sont donc actives qu'en présence de co-substrat. Ce sont elles qui sont impliquées dans le métabolisme bactérien de la thiamine chez les ruminants. Un grand nombre de molécules présentes dans les organismes vivants, acide nicotinique, delta-1-pyrroline, hypotaurine, cystine, proline, lysine, certaines bases non biologiques comme la pyridine ou la 3 - picoline, sont actives comme co-substrats. L'enzyme est inactivée par son substrat primaire, la thiamine, ou ses analogues structuraux et réactivée par son substrat secondaire ou cosubstrat. Les rapports de concentration entre substrat et co-substrat sont donc très importants dans le fonctionnement de l'enzyme. Une activité thiaminasique de type I a été retrouvée quasi systématiquement dans le contenu de rumen et les fèces d'animaux atteints de nécrose du cortex (Edwin et Jackman 1973) et également d'animaux ne présentant aucun signe clinique de déficience (Linklater et Dyson 1977).

Des tentatives d'isolement de germes producteurs de thiaminases à partir de contenu de rumen et de fèces de moutons atteints de NCC ont été réalisées par divers auteurs. Différents types de germes ont été isolés : un Bacillus producteur de thiaminases de type I, différent de Bacillus thiaminolyticus, Clostridium sporogenes (Morgan et Lawson 1974, Shreeve et Edwin 1974), B. thiaminolyticus et Clostridium sporogenes (Boyd et Walton 1977). Néanmoins ces germes ne sont 
Figure 4. Effet des thiaminases I et /l (d'après Harmeyer et Kollenkirchen 1989).<smiles>CCCCCCCCOCc1cnc(C)nc1N</smiles><smiles>Cc1ncc(C[n+]2csc(CCO)c2C)c(N)n1</smiles><smiles>Cc1ncc(CO)c(N)n1</smiles><smiles></smiles>

pas des hôtes habituels du rumen. Parmi 14 espèces habituelles du rumen, seule Megasphaera elsdenii présente une faible activité thiaminasique (Boyd 1985). L'affinité de la thiaminase de $M$. elsdenii pour les différents co-substrats connus est différente de celle des thiaminases retrouvées dans le rumen lors de NCC.

Si les thiaminases sont donc susceptibles de jouer un rôle important dans la genèse de la NCC, il ressort à l'évidence des données de la littérature que la présence de germes susceptibles de les produire dans le rumen est loin d'être constante, voire plutôt rare. On peut donc penser qu'elles ne sont pas responsables de la destruction ruminale de vitamine B1 observée lors d'un apport alimentaire très élevé de cette vitamine. Il est probable que la régulation de la production bactérienne nette de thiamine dans le rumen soit liée à des mécanismes de rétroinhibition puissants sur la synthèse et à des mécanismes de destruction de la thiamine intracellulaire non liés aux thiaminases.

\section{3 / Effet d'une supplémentation en thiamine de la ration}

\section{1 / Effet sur le métabolisme microbien dans le rumen}

En l'absence de toute connaissance précise sur le métabolisme microbien de la thiamine chez les différentes espèces composant l'écosystème rumen, il est possible de se demander si la synthèse bactérienne est dans tous les cas suffisante pour optimiser le métabolisme bactérien des espèces productrices de thiamine et pour couvrir le besoin des espèces non productrices. Quelques essais in vitro sur du jus de rumen provenant d'animaux nourris avec des régimes synthétiques montrent une action positive d'une adjonction de thiamine : augmentation de la synthèse protéique (Höller et al 1978, Kone 1978, Candau et Kone 1980), augmentation de la production d'AGCC lorsque la concentration de thiamine passe de 0,3 à 1,1 $\mu \mathrm{g} / \mathrm{l}$ (Höller et al 1978), modification des proportions relatives des AGCC avec aug- 
mentation du propionate ; de même en RUSITEC, la dégradation de la paille est significativement augmentée par l'adjonction d'un cocktail de vitamines du groupe B (Bouiller-Oudot et al 1988).

\section{2 / Effets zootechniques}

$\mathrm{Si}$, dans la plupart des cas, l'adjonction de thiamine au régime des ruminants ne provoque aucune modification de leurs performances, montrant par là même que la production intraruminale de cette vitamine et son absorption sont suffisantes pour assurer un apport optimum, certains essais montrent que l'adjonction de thiamine est susceptible d'augmenter les performances zootechniques des animaux. D'autres suggèrent un état subcarentiel sans manifestations pathologiques mais avec des performances détériorées. Des effets positifs sur la croissance ont pu être notés chez des bovins à l'engrais (Grigat et Mathison 1982). Chez le mouton, une amélioration de la production laitière des brebis et de la croissance des agneaux a pu être observée (McDonald 1982)

Ces effets positifs sont observés en général avec des régimes présentant des caractéristiques extrêmes (fourrages pauvres ou au contraire régimes très riches en concentrés). Il est difficile de savoir, dans ces cas, si l'effet positif d'une adjonction de thiamine est lié à une modification du métabolisme de la flore du rumen ou à un effet direct sur l'animal.

\section{4 / La nécrose du cortex chez les ruminants (NCC)}

La carence en thiamine chez les ruminants ne s'apparente pas aux carences vitaminiques classiques puisque les manifestations pathologiques observées ne sont pas liées à un défaut d'apport mais à une perturbation du métabolisme bactérien au niveau du rumen, associée ou non à des anomalies au niveau de l'absorption et de l'utilisation métabolique de cette vitamine.

La manifestation pathologique décrite à l'origine en Angleterre (Terlecki et Markson 1961) et identifiée en France six ans plus tard (Tournut et al 1967), est bien connue sous le nom de nécrose du cortex cérébral (NCC) ou polioencéphalomalacie (PEM) selon la nomenclature américaine. Si la cause exacte et le mécanisme d'apparition de cette maladie ne sont toujours pas connus, par contre on s'est très rapidement aperçu que l'administration de thiamine aux animaux atteints a un effet curatif remarquable.

\section{1 / Espèces affectées et conditions d'apparition}

La NCC est un syndrome connu dans le monde entier, pouvant atteindre toutes les espèces de ruminants. Elle est fréquente chez le mouton et les bovins, un peu moins chez la chèvre (Loew 1978, Smith 1979). On l'a même décrite chez le lama (Pugh 1993) et chez les ruminants sauvages comme les bisons ou les antilopes.

Le sexe ne joue aucun rôle dans l'apparition de la maladie. L'âge par contre est un facteur important : en Europe les animaux atteints sont essentiellement des jeunes, 2 à 7 mois pour les ovins, 3 à 30 mois pour les bovins. Par contre, dans les formes endémiques thiaminedépendantes observées en Côte d'Ivoire de 1989 à 1991 (Domenech et al 1994) ou en Inde (Lonkar et al 1993) les animaux atteints étaient des adultes.

La NCC peut être observée dans des conditions d'élevage extrêmement variables. En France, la plupart des cas signalés sont liés à une alimentation de type intensif, riche en concentrés, pauvre en cellulose et de faible fibrosité, favorisant l'apparition des états acidosiques ; les pulpes, la mélasse, les régimes à base d'ensilage de maïs sont également des facteurs favorisants. Inversement, dans les cas observés au pâturage, les niveaux d'apport peuvent être extrêmement variables. Par exemple, l'enzootie constatée en Côte d'Ivoire en 89-91 est apparue dans un contexte de sous-nutrition. D'une façon générale la NCC semble favorisée par des conditions d'alimentation extrêmes et aussi par des changements brusques de régimes.

\section{2 / Signes cliniques de la NCC}

\section{a / Symptômes}

La maladie peut évoluer de façon suraiguë aboutissant à la mort en 24-48 h ou plus lentement en $8-10$ jours. Après un épisode de diarrhée non obligatoire l'animal présente des troubles nerveux, une démarche mal assurée, des tremblements musculaires, des convulsions, des mouvements forcés. Très rapidement la station debout devient impossible, l'animal couché porte la tête en extension en arrière, il présente une cécité d'origine centrale sans lésion de l'œil (amaurose), une difficulté à mastiquer et à déglutir. L'issue mortelle est quasi systématique dans les cas suraigus non traités par la thiamine. Au contraire, les animaux atteints légèrement peuvent récupérer spontanément.

Trois types de modifications sanguines témoignent des perturbations biochimiques liées directement à la carence en B1 : l'augmentation de la pyruvicémie, de la lactacidémie et la diminution de l'activité transcétolasique érythrocytaire. Une imprécision quant à l'interprétation de ce dernier paramètre se dégage de la bibliographie, du fait de la diversité des méthodes de dosage existantes et de l'absence de standardisation chez les ruminants. Pour cette raison, certains auteurs ne prennent comme critère de modification de l'activité transcétolasique que "l'effet TPP", c'est-à-dire l'augmentation de l'activité transcétolasique observée lors d'une adjonction de thiamine pyrophosphate aux globules rouges faisant l'objet de la détermination. La déficience vitaminique ne peut être confirmée que si l'effet TPP est supérieur à 80\% (Jackman 1985). 


\section{b / Lésions}

Le cerveau présente des zones de 1 à $20 \mathrm{~mm}$ de diamètre d'aspect jaunâtre, présentant une fluorescence caractéristique sous lumière ultraviolette à $365 \mathrm{~nm}$, siégeant dans la substance grise, principalement au niveau des hémisphères cérébraux, plus rarement dans le cervelet ou les tubercules quadrijumeaux. Elles correspondent à des lésions de nécrose laminaire des neurones, associées à des zones d'œdème du tissu nerveux.

La teneur en vitamine B1 du cerveau mais également des autres tissus (foie, coeur) tombe à $20 \%$ de la valeur observée sur des animaux sains (Behrens et Höller 1977).

La NCC des ruminants se rapproche de l'encéphalopathie de Gayet-Wernicke observée chez l'homme recevant une nutrition parentérale de longue durée privée de vitamine $B 1$. Les lésions du cerveau observées sont liées aux déficits du métabolisme énergétique des cellules cérébrales. La non utilisation du pyruvate, de l'alphacétoglutarate provoque une diminution du taux d'ATP dans le cerveau entraînant une diminution de l'activité de la $\mathrm{Na}-\mathrm{K}$ ATPase, une détérioration du transport ionique à travers les membranes et un oedème des astrocytes. Chez les monogastriques au contraire, ou dans le béribéri humain, on observe surtout une polynévrite périphérique se traduisant par une détérioration de la conduction nerveuse.

\section{3 / Traitement}

Dans la plupart des cas, l'administration de vitamine $\mathrm{B} 1$ aux animaux atteints de NCC permet une rémission rapide et souvent complète des signes cliniques et la survie de l'animal. Le succès de cette thérapeutique permet du même coup de confirmer le diagnostic de NCC. Pour avoir une efficacité thérapeutique les quantités de thiamine administrées doivent être très élevées, de l'ordre de $500 \mathrm{mg}$ pour des moutons et de $2 \mathrm{~g}$ pour des bovins, ce qui représente environ 100 fois les quantités journalières nécessaires pour couvrir les besoins normaux.

\section{4 / Les tentatives de reproduction expérimentale de la NCC}

La NCC a pu être reproduite expérimentalement soit en utilisant des analogues structuraux de la thiamine sans pouvoir vitaminique, soit en introduisant des thiaminases dans le régime alimentaire, soit en utilisant des régimes synthétiques.

Parmi les analogues structuraux de la thiamine ayant un effet antivitaminique, amprolium, oxythiamine, pyrithiamine, seul l'amprolium permet une reproduction expérimentale satisfaisante chez le mouton (Espinasse et al 1971, Loew et Dunlop 1972, Chahar et al 1993) à condition d'en administrer des quantités considérables, de l'ordre de 800 à $1000 \mathrm{mg}$ par kilo de poids corporel pendant 30 à 80 jours. Dans ces conditions, l'ordre de grandeur du rapport entre l'amprolium administré et la thiamine synthétisée journellement dans le rumen est de 104. Chez le veau, le syndrome a pu être reproduit avec des quantités d'amprolium dix fois plus faibles mais sur des animaux non sevrés (Markson et al 1972).

La NCC a pu également être reproduite expérimentalement chez le mouton par administration continue pendant 25 à 40 jours de rhizome de fougère aigle très riche en thiaminase (Evans et al 1975).

La troisième voie de reproduction est constituée par les régimes synthétiques dépourvus de thiamine, soit chez les ruminants allaités n'ayant pas encore une synthèse ruminale de vitamines (Thornber et al 1981), soit sur des agneaux sevrés (Naga et al 1975). La NCC a pu également être reproduite en quelques jours avec une alimentation entérale chez le mouton (Lusby et Brent 1972).

\section{5 / Mécanisme d'apparition de la NCC}

\section{a / L'hypothèse «thiaminases»}

C'est l'hypothèse la plus classique (Edwin et al 1979). Dans la plupart des cas confirmés de NCC, en effet, on retrouve une activité thiaminasique dans le contenu de rumen et également le plus souvent au niveau des fèces. Cette hypothèse est confortée par la reproduction expérimentale de la NCC chez le mouton avec la thiaminase I de la fougère aigle. Elle est cependant mise à l'épreuve par un certain nombre de faits contradictoires. On retrouve en effet une fois sur quatre une activité thiaminasique positive dans le contenu de rumen d'animaux parfaitement sains. L'administration continue pendant plusieurs semaines à des agneaux normaux et gnotobiotiques d'une souche de Clostridium sporogenes thiaminase + isolée à partir d'animaux morts de NCC n'a pas permis de reproduire le syndrome malgré la persistance d'une activité thiaminasique intense dans le rumen (Cushnie et al 1979). Il ressort de l'ensemble de la littérature que si la présence d'une activité thiaminasique dans le rumen est le plus souvent la règle dans les cas spontanés de NCC, elle ne constitue pas une condition suffisante pour déclencher la NCC. La présence d'une activité thiaminasique sur des animaux sains n'est d'ailleurs pas absolument contradictoire puisqu'on peut l'observer sur des animaux sains mais présentant un état de nutrition vitaminique B1 non optimal (Thomas 1986). On peut concevoir que si le bilan synthèse microbienne de B1 - destruction par les thiaminases reste positif, l'animal ne développe pas de signe de déficience. Il est malheureusement prématuré d'en juger d'après les résultats de la littérature, les deux termes du bilan précédent n'ayant pratiquement jamais été mesurés simultanément. Les données initiales concernant l'activité thiaminasique du rumen sont semi-quantitatives et dans les premières publications la méthode de dosage utilisée (Edwin et Jackman 1974) est critiquable, les concentrations de substrats et de co-substrats utilisées n'optimisant pas la cinétique enzymatique.

Dans la plupart des cas, on ne connait pas l'origine des thiaminases. Il y a peu de chances pour qu'elles soient apportées directement par
La surproduction de thiaminase dans le rumen existe en général dans les cas de NCC, mais elle ne suffit pas à déclencher la pathologie. 
Figure 5. Lésions cérébrales observées sur un mouton soumis pendant 4 mois à un régime semi-synthétique hypersoufré $(0,6 \% \mathrm{~S})$ et dépourvu de thiamine. Les flèches indiquent des zones de nécrose avec disparition de la substance grise du cortex (cliché ENVL/Jean-Blain - Létourneau).

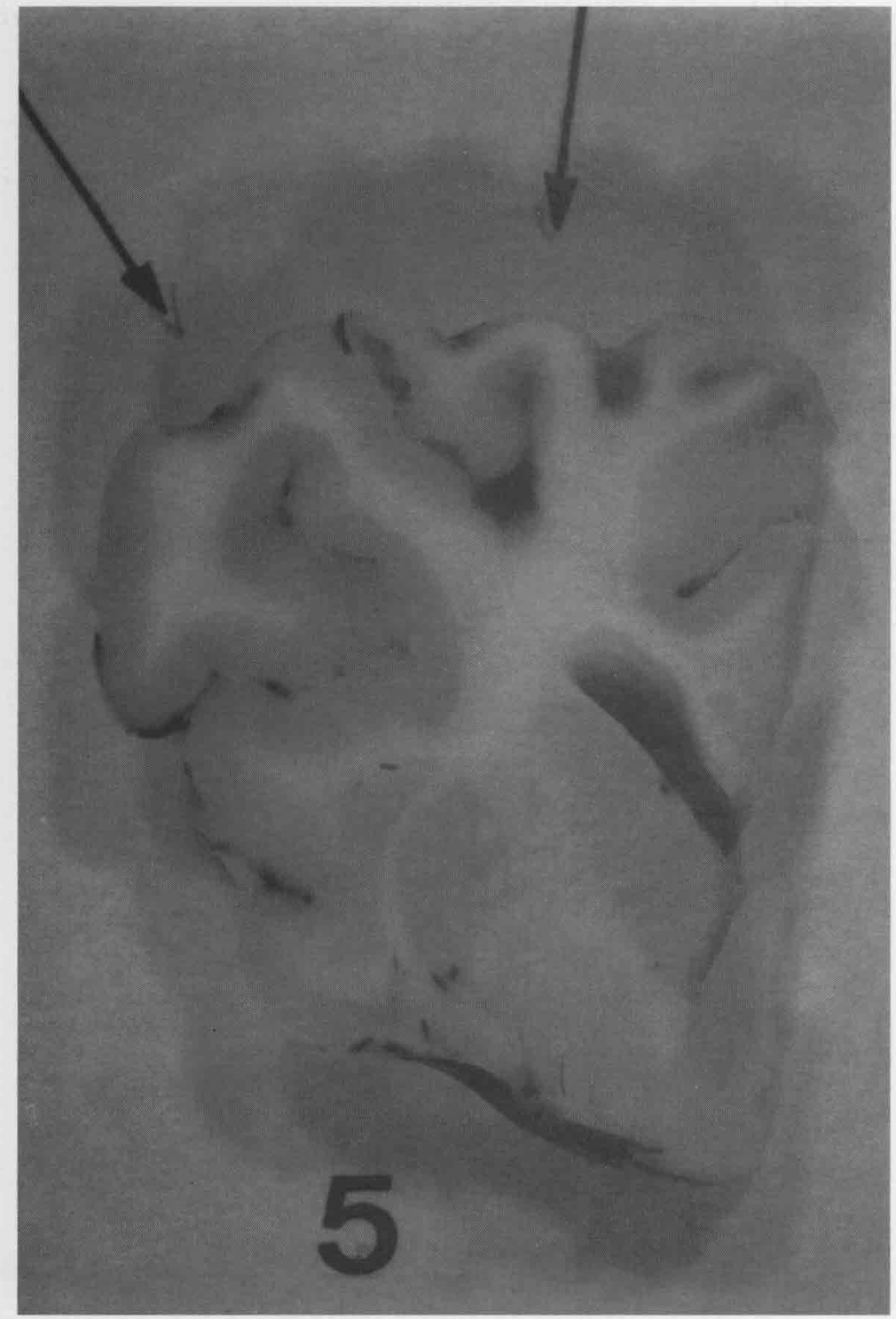

les aliments, l'activité thiaminolytique mise en évidence dans certains aliments in vitro ne se retrouvant pas in vivo. Par ailleurs, les principaux genres constituant l'ossature de l'écosystème microbien ruminal n'ont pas ou très peu d'activité thiaminasique dans les conditions normales. Il semble que les micro-organismes producteurs de thiaminases soient donc des germes de passage introduits dans le rumen à partir de l'environnement. Des travaux récemment réalisés en Australie accréditent l'intervention des thiaminases dans la genèse à la fois de la NCC clinique et d'un état de subcarence en thiamine se traduisant par une baisse des taux de thiamine dans les tissus, de la transcétolase érythrocytaire et des baisses de performances. Ces états carentiels apparaissent soit à la suite de stress liés au transport maritime des moutons (Thomas et al 1990) soit sur de très jeunes agneaux. Dans ce dernier cas, l'intervention de Bacillus thiaminolyticus a pu être prouvée. L'apparition d'une activité thiaminasique élevée dans le rumen serait donc une condition nécessaire mais non suffisante pour faire apparaitre la NCC. La persistance d'une activité thiaminasique élevée dans des conditions favorisant la destruction de la thiamine paraît nécessaire, ces conditions incluent l'ingestion et la synthèse ruminale d'une quantité faible de thiamine et la présence abondante de co-substrats inhibant la thiaminase.

La partie la moins élaborée de l'hypothèse "thiaminases" réside dans la connaissance imprécise des facteurs susceptibles de favoriser l'installation des bactéries sécrétant des thiaminases. Si des facteurs comme les régimes concentrés pauvres en lest, les états d'acidose, l'usage d'azote non protéique, les changements brutaux de régime sont considérés comme facteurs de risque majeurs, la manipulation expérimentale de ces facteurs sur des animaux sains n'a jamais réussi à faire apparaître un état de déficience en vitamine B1 et, a fortiori, de symptôme de NCC. Un régime acidogène, riche en concentrés chez l'agneau ne fait pas apparaître d'activité thiaminasique (Sapienza et Brent 1974). La modélisation in vitro en fermenteur continu d'un état d'acidose chronique ne fait pas diminuer la synthèse de B1 et ne fait pas apparaître d'activité thiaminasique (Alves de Oliveira et al 1994).

\section{b / L'hypothèse «excès de soufre»}

Cette voie de recherche se développe actuellement parallèlement à des enquêtes épidémiologiques sur la NCC aux USA (Raisbeck 1982) et au Canada (Olkowski et al 1991a, Hamlen et al 1993) mettant en relation l'apparition de la NCC avec un apport excédentaire de sulfates consécutif à un abreuvement avec des eaux sulfatées (2000 à $7000 \mathrm{mg} / \mathrm{l}$ ) ou à l'incorporation de sulfates dans la ration à des taux égaux ou supérieurs à $2 \%$ à titre d'additif limitant l'ingestion. Ces constatations ne sont d'ailleurs pas entièrement nouvelles puisque, en Europe, l'emploi de pulpes renfermant du sulfate d'aluminium utilisé comme agent de pressage a aussi été incriminé dans la genèse de la NCC. Les travaux expérimentaux développés par les Canadiens à la suite des investigations de terrain ont montré qu'il est possible de reproduire, chez le jeune agneau, en 3 à 7 semaines un syndrome nerveux tout à fait comparable, sinon identique sur le plan clinique et lésionnel à la NCC classique, par administration d'un régime renfermant $0,63 \%$ de soufre (niveau normal $0,15-0,25 \%$ ) (Gooneratne et al 1989a, Rousseaux et al 1991). Des cas de NCC ont été également obtenus sur des génisses à l'engrais recevant un régime expérimental riche en soufre (Gooneratne et al 1989b).

\section{Relation avec la thiamine}

La NCC déclenchée par un régime excédentaire en soufre (figure 5) est-elle en relation comme la NCC classique avec un état de déficience en thiamine ou s'agit il de deux affec- 
tions totalement différentes mais se traduisant par des manifestations cliniques voisines, voire identiques?

Une enquête réalisée sur 50 exploitations bovines canadiennes (Olkowski et al 1991a) semblait tout d'abord accréditer la liaison soufre thiamine, les animaux consommant des eaux sulfatées ayant un taux sanguin de thiamine significativement plus bas que ceux consommant une eau à faible teneur en sulfates. En fait, d'une part les teneurs sanguines relevées au cours de l'enquête sont extrêmement dispersées, la teneur moyenne du sang total en thiamine chez les animaux buvant des eaux sulfatées est de $37 \mu \mathrm{g} / \mathrm{l}$, très supérieure à la valeur seuil de $25 \mu \mathrm{g} / \mathrm{l}$ considérée comme normale. On sait par ailleurs que le taux sanguin de thiamine n'a qu'une valeur limitée pour apprécier le niveau de nutrition relatif à cette vitamine, compte tenu des fluctuations très grandes observées, quel que soit le régime. Les expérimentations ultérieures réalisées par les mêmes auteurs ont montré qu'il n'y avait aucune relation entre le taux de thiamine dans le sang et le niveau d'apport de soufre. Par ailleurs la teneur en thiamine du foie et du cerveau ainsi que l'activité transcétolasique ne sont pas modifiées chez les animaux recevant un régime à teneur excédentaire en soufre, que ces animaux développent ou non un syndrome NCC. Enfin, l'administration de vitamine $\mathrm{B} 1$ à haute dose n'a dans ces cas pas d'effet curatif. Il s'agit donc bien ici d'un trouble différent de la NCC classique thiamine dépendante, très probablement lié à une intoxication par les sulfures produits dans le rumen par réduction des sulfates. Cette intoxication par les sulfures a été reproduite expérimentalement chez l'agneau (McAllister et al 1992) par infusion répétée de sulfures directement dans le rumen à raison de $75 \mathrm{mg}$ de S par kilo de poids métabolique. On produit ainsi des lésions nerveuses de nécrose, de nature analogue à celles qui sont observées dans la maladie naturelle. L'induction de ces lésions ne s'accompagne d'aucune modification des paramètres de la nutrition thiaminique, elles ne régressent pas lors d'administration de vitamine $\mathrm{B} 1$.

L'absence de détérioration de la nutrition vitaminique $B 1$ sous l'effet d'un excès de soufre n'exclut pas pour autant toute interaction entre ces deux nutriments. Par ailleurs l'action pharmacodynamique de la vitamine B1 administrée à dose très élevée peut expliquer son action positive correctrice dans certains troubles observés lors d'excès de soufre, par exemple un effet positif sur l'appétit ou sur la conductibilité nerveuse.

\section{c / Interactions éventuelles soufre vitamine $\mathrm{B1}, \mathrm{Cu}, \mathrm{Mo}$}

Un régime à haute teneur en soufre associé à un apport en cuivre marginal ou rendu insuffisant par un excès de molybdène, provoque une diminution de la teneur en thiamine du sang et du liquide duodénal (Olkowski et al $1991 b)$. Les variations observées sont cepen- dant faibles, elles ne sont pas retrouvées au niveau du foie, du rein et du cerveau et elles restent dans des limites considérées comme normales. Cette interaction n'est donc pas clairement établie.

\section{d / Interaction plomb / thiamine}

La thiamine administrée par voie parentérale et à dose élevée diminue la teneur en plomb dans le foie et les reins chez les animaux ayant ingéré des doses toxiques de plomb, et agit également comme antidote (Bratton et al 1981)

\section{Conclusion}

Dans l'état actuel des connaissances, la NCC des ruminants apparaît comme la manifestation clinique commune de deux entités pathologiques distinctes, la première, la plus anciennement reconnue, étant reliée à une perturbation de la nutrition thiaminique dont le mécanisme intime nous échappe encore, l'autre étant reliée à un apport excédentaire de soufre dans la ration. S'il est prématuré d'affirmer une totale absence d'interactions entre ces deux pathologies, du moins le niveau d'interaction, s'il existe, est faible.

En ce qui concerne la NCC thiamine dépendante, son apparition reste vraisemblablement liée à l'apparition d'une activité thiaminasique au niveau du tube digestif, mais pour que cette activité thiaminasique aboutisse à des manifestations cliniques, il faut qu'elle s'exerce avec une intensité suffisante et pendant un temps assez long. En soi elle n'est pas suffisante pour déclencher l'apparition des manifestations cliniques de la NCC. Les conditions nutritionnelles aptes à créer cette activité ou à permettre l'implantation des germes thiaminolytiques dans le rumen sont encore inconnues.

L'exploration biochimique de l'état de nutrition thiaminique permet de mettre en évidence des états de subcarence non révélés par des symptômes caractéristiques mais seulement par des déficits zootechniques.

Par ailleurs, s'il a été abondamment prouvé que dans la plupart des cas la supplémentation en thiamine de la ration des ruminants est inutile, dans certains régimes extrêmes à base de fourrages pauvres ou inversement de régimes très riches en concentrés, une supplémentation en thiamine peut contribuer à optimiser les processus fermentaires.

Les facteurs régulant la synthèse et la destruction microbienne de thiamine dans le rumen restent encore très imparfaitement connus. Par ailleurs, si dans la majorité des cas, la synthèse de vitamine $B 1$ est suffisante pour prévenir tout signe de carence, il n'est pas certain, compte tenu des processus de destruction microbiens de la thiamine dans le tube digestif, que l'apport ainsi réalisé permettre toujours d'optimiser le fonctionnement de l'organisme.

\section{Un régime excédentaire en soufre déclenche une NCC, mais d'étiologie différente, et l'apport de thiamine n'a pas d'effet curatif.}




\section{Références bibliographiques}

Agrawala I.P., Huffmman c.f., Luecke r.w., Duncan c.w., 1953. A quantitative study of rumen synthesis in the bovine on natural and purified rations. J.Nutr., 49, 631-638.

Alves de Oliveira L., Durix A., Bony S., Jean-Blain C., 1994. Etude en RUSITEC de l'influence du pH sur la synthèse de thiamine. Ann. Zootech. (Jour. Nutr. Herb. Paris 1992) sous presse.

Behrens H., Höller H., 1977. Thiamingelhalte in Lebern und Gehirnen von Schafen mit Cerebralnekrose, Listeriose und anderen Erkrankungen. Dtsch. Tierärztl. Wschr., 84, 293-332.

Barschdorf D., 1985. Gastrointestinale Bilanz von Thiamin (Vit.B1) bei Schafen mit diätetisch bedingter Phosphordepletion. Thes. Doc. Vet. Tierärztl. Hochsch. Hannover.

Bechdel S.I., Honeywell H.E., Dutcher R.A., Knutson M.H., 1928. Synthesis of vitamin B in the rumen of the cow. J. Biol. Chem., 80, 231-238.

Bick S., Breves G., Höller H., 1978. Nettosynthese von mikrobiellem Protein und Thiamin im Panseninhalt eiweissfrei ernährter Schafe bei unterschiedlichen Substrat-und Schwefelkonzentrationen in vitro. Zeitz. Tierphysiol. Tierernährg; Futtermittelk., 41, 8-17.

Bouiller-Oudot M., Belfadla A., Candau M., 1988. Influence de l'addition de vitamines du groupe $B$ sur la dégradation des constituants pariétaux (paillefoin-pulpes) par les microorganismes du rumen en fermenteur semi-continu. Reprod. Nutr. Dévelop., $28,153-154$.

Boyd J.W., 1985. Studies on thiaminase I activity in ruminant faeces and rumen bacteria. J. Agric. Sci., Camb., 104, 637-642.

Boyd J.W., Walton J.R., 1977. Cerebrocortical necrosis in ruminants : an attempt to identify the source of thiaminase in affected animals. J. Comp. Path., 87, 581-589.

Bratton G.R., Zmudzki J., Kincaid N., Joyce J., 1981. Thiamin as treatment of lead poisoning. Modern Vet. Pract., 62, 441-446.

Breves G., Brandt M., Hoeller H., Rohr K., 1981 Flow of thiamin to the duodenum in dairy cows fed different rations. J. Agric. Sci., 96, 587-591.

Breves G., Schmitt R., Höller H., 1982. Thiamin compartment models in healthy sheep. Internat. J. Vit. Nutr. Res., 52, 3-8.

Candau M., Kone L., 1980. Influence de la thiamine sur la protéosynthèse bactérienne chez le mouton. Reprod. Nutr. Dévelop., 20, 1695-1699.

Candau M., Massengo J., 1982. Evidence of a thiamine deficiency in sheep fed maize silage. Ann. Rech. Vét., 13, 329-340.

Chahar A., Yadav J.S., Sharma S.N., Vyas U.K., 1993. Experimental studies on polioencephalomalacia (cerebro-cortical necrosis) in sheep induced by Amprolium. Indian Vet. J., 70, 411-413.

Cushnie G.H., Richardson A.J., Lawson W.J., Sharman A.M., 1979. Cerebrocortical necrosis in ruminants : Effects of thiaminase type 1-producing Clostridium sporogenes in lambs. Vet. Rec., 24, 480-482.
Domenech J., Wyers M., Braun J.P., Formenty P., 1993. The ovine nervous syndrome in Côte d'Ivoire polioencephalomalacia associated with thiamine deficiency. Rev. Elev. Méd. vét. Pays trop., 46, sous presse.

Edwin E.E., Jackman R., 1973. Ruminal thiaminase and tissue thiamine in cerebrocortical. Vet. Rec., 92, 640-641.

Edwin E.E., Jackman R., 1974. A rapid radioactive method for determination of thiaminase activity and its use in the diagnosis of cerebrocortical necrosis in sheep and cattle. J. Sci. Fd Agric., 25, 357-368.

Edwin E.E., Markson L.M., Shreeve J., Jackman R., Caroll P.J., 1979. Diagnostic aspects of cerebrocortical necrosis. Vet. Rec., 6, 4-8.

Egi Y., Koyama S., Shioda T., Yamada K., Kawasaki T., 1992. Identification, purification and reconstitution of thiamin metabolizing enzymes in human red blood cells. Biochim. Biophys. Acta, 1160, 171-178.

El Hindi H., 1977. In vitro Untersuchungen zur Umsatz von Thiamin im Panseninhalt eiweissfrei ernährter Schafe. Thesis D.M.Vet. Tierärztliche Hochschule Hannover (Germany)

Espinasse J., Redon P., Sinha R.P., 1971. Reproduction expérimentale de la nécrose du cortex cerebral chez le mouton. Rev. Méd. Vét, 122, 529-545.

Evans W.C., Evans I.A., Humphreys D.J., Lewin B., Davies W.E.J., Axford R.F.E., 1975. Induction of thiamine deficiency in sheep with lesions similar to those of cerebrocortical necrosis. J. Comp. Pathol., 85, 253-267.

Fujita A., 1954. Thiaminase. Adv.Enzymol., 15, 389421.

Gooneratne S.R., Olkowski A.A., Christensen D.A., 1989a. Sulfur-Induced Polioencephalomalacia in Sheep : Some biochemical Changes. Can. J. Vet. Res., 53, 462-467.

Gooneratne S.R., Olkowski A.A., Klemmer R.G, Kessler G.A., Christensen D.A., 1989b. High sulfur related thiamine deficiency in cattle : A field study. Can. Vet. J., 30, 139-146.

Grigat G.A., Mathison G.W., 1982. Thiamin supplementation of all-concentrate diet for feedlot steers. Can. J. Anim. Sci., 62, 807-819.

Haas R.H., 1988. Thiamin and the brain. Ann. Rev. Nutr., 8, 483-515.

Hamlen H., Clark E., Janzen E., 1993. Polioencephalomalacia in cattle consuming water with elevated sodium sulfate levels : A herd investigation. Can. Vet. J., 34, 153-158.

Harmeyer J., Kollenkirchen U., 1989. Thiamin and niacin in ruminant nutrition. Nutr. Res. Rev., 2, 201-225.

Haven T.R., Caldwell D.R., Jensen R., 1983. Role of predominant rumen bacteria in the cause of polioencephalomalacia (cerebrocortical necrosis) in cattle. Am. J. Vet. Res., 44, 1451-1455. 
Hill J.H., Rammell C.G., Forbes S, 1988. Blood thiamine levels in normal cattle at pasture. N. Z. vet. J., 36, 49-50.

Höller H., Schaller K., Behrens H., 1976. Untersuchungen über die Vitamin B1inaktivierende Wirkung in vitro von pflanzlichen Futtermitteln. Dtsch. Tieraertl. Wochenschr., 83 $108-111$

Höller H., Fecke M., Schaller K., 1977. Permeability to thiamin of the sheep rumen wall in vitro. J. Anim. Sci., 44, 158-163.

Höller H., El Hindi H., Breves G., 1978. Einfluss von Thiamin (vitamin B1) auf mikrobielles Wachstum und Bildung von flüchtlichen Fettsäuren in-vitro im Pansensaft proteinfrei ernährter Schafe. Dtsch. Tierärztl. Wschr., 85, 200-202.

Höller H., Buchhop K., Breves G., 1979 Untersuchungen zur Passage von Thiamin (Vit B1) durch die Pansenwand von Schafen in vivo. Zbl. Vet. Med. A, 26, 841-846.

Höller H., Hübel U., Breves G., 1982. Untersuchungen zur Thiaminresorption aus dem Dickdarm von Schafen mit Hilfe der Colonperfusion. Zbl. Vet. Med. A, $29,619-627$

Inglis D.M., Walker H.F., Mian D.H., Mnangome W., 1989. Comparison of three methods of thiamine supplementation by measurement of urinary thiamine excretion in sheep. Res. vet. Sci., 47, 225-230.

Jackman R., 1985. The diagnosis of CCN and thiamine deficiency in ruminants. Vet. Annu., 25, 71-77.

Kirchgessner M., Roth-Maier D.A., Paulicks R. 1988. B-Vitamine (Thiamin, Vitamine B6, Vitamine B12 und Pantothensäure) in der Kuhmilch be Energie-oder Proteinmangel und anschliessender Realimentation. J.Anim.Physiol.a.Anim.Nutr. 59 , $255-262$

Kone L. 1978.Influence de la thiamine sur la protéosynthèse bactérienne chez le mouton. Thes. Doc. Ing. Inst.Polyt.TOULOUSE.

Lebzien P., Rohr K., Breves G., Höller H., 1986. Untersuchungen über den Einfluss von Rumensin (Monensin-Natrium) auf die Stickstoffumsetzungen und die Thiaminnettosynthese in den Vormägen von Wiederkäuern. J. Anim. Physiol. a. Anim. Nutr. 55 , $177-186$

Leichter J., Joslyn M.A. 1969. Kinetics of thiamin cleavage by sulphite. Biochem. J., 113, 611-615.

Linklater K.A., Dyson D.A. 1977.Faecal thiaminase in clinically normal sheep associated with outbreaks of polioencephalomalacia. Res. Vet. Sc., 22, 308-312.

Loew F.M. 1978. Effect of nutrient deficiencies in animals : thiamin, in CRC Handbook Series in Nutr. Food.RECHCIGL M. Ed. Section E Nutritional disorders $3-23$

Loew F.M., Dunlop R.H. 1972.Induction of thiamine inadequacy and polioencephalomalacia in adult sheep with Amprolium. Am. J. Vet. Res., 33, 21952205.

Loew F.M., Bettany J.M., Halifax C.E. 1975. Apparent thiamin status of cattle and its relationship to polioencephalomalacia. Can. J. comp. Med., 39, 291 295
Lonkar P.S.,Sharma S.N.,Yadav J.S.,Prasad M.C., 1993. Epidemiology of cerebrocortical necrosis in goats. Indian Vet. J., 70, 873-875.

Lusby K.S., Brent B.E., 1972. An experimental model for polioencephalomalacia. J. Anim.Sci.35, 270.

Markson L.M., Lewis G., Terlecki S., Edwin E.E. Ford J.E., 1972.The etiology of cerebrocortical necrosis:the effects of administering antimetabolites of thiamine to preruminants calves. Br. Vet. J., 128 , 488-499.

Massengo J. 1977. Recherches sur la carence en thiamine chez le mouton reçevant un régime d'ensilage de maïs. Th. Doc. Ing. Inst. Polyt. Toulouse.

McAllister M.M., Gould D.H., Hamar D.W., 1992 Sulphide-induced Polioencephalomalacia in lambs. J. Comp. Pathol., 106, 267-278.

McDonald J.W. 1982.Mortality and illthrift associated with thiamine deficiency in lambs. Aust. Vet. J. 58, 212-213.

McElroy L.W., Goss H. 1941.A quantitative study of vitamins in the rumen content of sheep and cows fed vitamin-low diets. III Thiamine. J. Nutr., 21, 163-173.

Miller B.L., Meiske J.C., Goodrich R.D., 1986. Effects of grain source and concentrate level on Bvitamin production and absorption in steers. J. Anim. Sci., 62, 473-483.

Morgan K.T. 1974.Thiaminase type 1-producing bacilli and ovine polioencephalomalacia. Vet. Rec. $95,361-63$

Moseley R.H., Vashi P.G., Jarose S.M., Dickinson C.J. Permoad P., 1992. Thiamine transport by basolateral rat liver plasma membrane vesicles. Gastroenter. 103, 1056-1065.

Naga M.A., Harmeyer J.H., Holler H., Scaller K., 1975.Suspected "B»vitamin deficiency of sheep fed a protein-free urea containing purified diet. J. Anim. Sci., 40, 1192-1197.

Newell P.C., Tucker R.G., 1966. The control mechanism of thiamin biosynthesis. Biochem. J., 100, $517-524$

Olkowski A.A., 1992.Role of thiamine status in sulphur induced polioencephalomalacia in sheep. Res. Vet. Sci., 52, 78-85.

Olkowski A.A., Rousseaux C.G., Christensen D.A 1991. Association of sulfate-water and blood thiamine concentration in beef cattle : Field studies. Can. J. Anim. Sci., 71, 825-832.

Olkowski A.A., Gooneratne S.R., Christensen D.A. 1991. The effects of copper status on thiamine metabolism in sheep fed high sulfur diet. Can. J. Anim. Sci. 71, 813-824

Olkowski A.A., Laarveld B., Patience J.F., Francis S.I., Christensen D.A., 1993.The effect of sulphate on thiamine-destroying activity in rumen content cultures in-vitro. Internat. J. Vit. Nutr. Res. 63, 38-44.

Pugh D.G., Diplomate, 1993. Polioencephalomalacia in a llama herd. Equine Pract., 15, 24-26.

Raisbeck M.F., 1982. Is polioencephalomalacia associated with high sulfates diets? J. Am Vet Med Assoc, 180, 1303-1305. 
Rousseaux C.G., Olkowski A.A., Chauvet A., Gooneratne S.R., Christensen D.A., 1991. Ovine Polioencephalomalacia Associated with Dietary Sulfur Intake. J.Vet.Med. A.38, 229-239.

Sapienza D.A., Brent B.E., 1974. Ruminal thiaminase vs.concentrate adapatation.J.An.Sc. 39, 251.

Schaller K., Brammertz-Buchen Chr., Brammertz K.H., Höller H.,1977. Untersuchungen zur biologischen Wirksamkeit sogenannter Thiaminantagonisten bei Ratten mit suboptimaler Thiaminversorgung. Z. Tierphysiol., Tierernähr., Futtermittelkde, 38, 65-74.

Shreeve J.E., Edwin E.E. 1974.Thiaminaseproducing strains of Cl.sporogenes associated with outbreaks of cerebrocortical necrosis.Vet.Rec.13, 330

Smith M.C., 1979. Polioencephalomalacia in goats. J.Am.Vet.Med.Assoc, 174, 1328-1332.

Steinberg W., Kaufmann W., Hagemeister H., 1977. Messungen zur Bestimmung der Resorption von Thiamin in den Vormägen und Darm von Milchkühen J. Tierphysiol.Tierernähr, Futtermittelk. 39, 282-288.

Terlecki S., Markson L.M., 1961. Cerebrocortical necrosis in cattle and sheep.Vet.Rec., 73, 23-27.
Thomas K.W., 1986.The effect of thiaminase-induced subclinical thiamine deficiency on growth of weaner sheep. Vet. Res. Comm., 10, 125-141.

Thomas KW., Kelly AP., Beers PT., Brennan RG., 1990. Thiamine deficiency in sheep exported live by sea. Austr. Vet. J., 67, 215-218.

Thornber E.J., Dunlop R.H., Gawthorne J.M., Huxtable C.R.1981. Induced thiamin deficiency in lambs.Austr.Vet.J., 57, 21-25.

Tournut J., Labie Ch., Espinasse J., 1967. Identification en France de la "Nécrose du Cortex Cérébral»(NCC) chez plusieurs espèces de ruminants. Rev. Méd. Vét., 118, 883-896.

Virtanen A.I.1967. The production of milk on protein-free rations in "Urea as a protein supplement" BRIGGS M.H. Ed. Pergamon press 1967.

Ziporin Z.Z., Nunes W.T., Powell R.C., Waring P.P., Sauberlich H.E., 1985. Excretion of thiamine and its metabolites in the urine of young adult males receiving restricted intakes of vitamin. J. Nutr., 85, 287-296.

\begin{abstract}
Physio-pathological aspects of thiamin (vitamin B1) in ruminants.

This review aims first to provide specific information on microbial synthesis, the absorption and the metabolism of vitamin $B 1$ in healthy ruminants, and on the influence of low thiamin production on rumen microbial activity. In the second part the authors discuss the etiology and the pathogenesis of cerebrocortical necrosis in the light of the most recent data. The factors involved in the microbial synthesis and catabolism of thiamin under usual feeding conditions are well known; however factors liable to disturb the net production or absorption of thiamin in the digestive tract under low- or overfeeding conditions, or in the case of inbalanced diets, are not well understood.
\end{abstract}

Summary
Classical pathogenesis of cerebrocortical necrosis involves the ruminal overproduction of thiaminases $I$. This theory is not entirely satisfactory, since the normal rumen microflora has a low thiaminase I activity and since factors which allow the establishment of thiaminase producing bacteria are not known. Excess of sulphur in the diet also induces typical signs of cerebrocortical necrosis but its relation to a thiamin deficiency is not clear. It is more likely that we are dealing with two independant etiologies which are expressed by the same clinical syndrome.

JEAN-BLAIN C., ALVES de OLIVEIRA L., 1994. Aspects physio-pathologiques de la thiamine (vitamine $\mathrm{BI}$ ) chez les ruminants. INRA Prod. Anim., 7 (2), 71-84 